\title{
Proton Pump Inhibitors Are Associated With Minimal and Overt Hepatic Encephalopathy and Increased Mortality in Patients With Cirrhosis
}

Silvia Nardelli, ${ }^{1}$ Stefania Gioia, ${ }^{1}$ Lorenzo Ridola, ${ }^{1}$ Alessio Farcomeni ${ }^{(D)},{ }^{2}$ Manuela Merli, ${ }^{1}$ and Oliviero Riggio ${ }^{1}$

\begin{abstract}
Minimal hepatic encephalopathy (MHE) is a subclinical cognitive impairment frequently observable in patients with cirrhosis. Proton pump inhibitors (PPIs) can contribute to small-bowel bacterial overgrowth, but no study has investigated the link between PPIs and MHE. We investigated the relationship between MHE and PPI use as well as the role of PPI use in the development of overt HE and survival. Consecutive patients with cirrhosis $(n=310)$ were included in the study and followed up for $14.1 \pm 12.3$ months. At entry, MHE was diagnosed when the Psychometric Hepatic Encephalopathy Score was $\leq-4$. Data were analyzed by logistic regression for the factors associated with MHE and by time-related models for overt HE development and survival. At inclusion, 131 out of 310 patients with cirrhosis $(42 \%)$ were affected by MHE. One hundred and twenty-five patients $(40 \%)$ were using PPIs. The variables independently associated with the presence of MHE were PPI use, previous overt HE, low albumin, low sodium, and age. During follow-up, the development of overt HE was higher $(64 \%$ versus $25 \%, P<0.001)$ and overall survival lower $(41 \%$ versus $81 \%, P<0.001)$ in PPI users than in nonusers. Variables independently associated with the development of overt HE were PPIs, history of overt HE, low albumin, MHE, and age, while variables independently associated with mortality were PPIs, development of overt HE, Model for End-Stage Liver Disease score, low sodium, and age. Conclusion: The study identifies a potentially removable factor associated with the presence of MHE and related to the development of overt $\mathrm{HE}$ and survival in patients with liver cirrhosis. (Hepatology 2019;0:1-10).
\end{abstract}

$\mathrm{O}$ vert hepatic encephalopathy (HE) is a spectrum of neuropsychiatric alterations, ranging from mild confusion to coma, which are observable in patients with advanced cirrhosis or portosystemic shunts. ${ }^{(1)}$ The pathogenesis of HE is not completely understood, but a relationship between $\mathrm{HE}$ and gut bacteria has been suggested for a long time. ${ }^{(2)}$ In fact, gut bacteria are responsible for the formation and release of products such as ammonia and endotoxins, implicated in the pathophysiology of HE. ${ }^{(3)}$ Moreover, treatments able to modify the gut flora such as antibiotics, ${ }^{(4-6)}$ disaccharides, ${ }^{(6,7)}$ probiotics, ${ }^{(7-9)}$ and fecal transplantation ${ }^{(10,11)}$ have been shown to have a beneficial effect on HE. Thus, factors able to modify the gut microbiota may affect HE. Proton pump inhibitors (PPIs) are strong gastric acid suppressants, widely prescribed, often inappropriately, in patients with chronic liver disease, ${ }^{(12)}$ which cause quantitative and qualitative alterations in gut microbiota. ${ }^{(13-16)}$ In fact, PPIs can directly target the proton pumps of the bacteria ${ }^{(17)}$ or affect the microenvironment by changing the $\mathrm{pH}$ within

Abbreviations: AUROC, area under the receiver operating characteristic curve; CI, confidence interval; HE, hepatic encephalopathy; HR, hazard ratio; MELD, Model for End-Stage Liver Disease; MHE, minimal HE; PHES, Psychometric Hepatic Encephalopathy Score; PPI, proton pump inhibitor.

Received June 18, 2018; accepted October 2, 2018.

(C) 2018 by the American Association for the Study of Liver Diseases.

View this article online at wileyonlinelibrary.com.

DOI 10.1002/hep.30304

Potential conflict of interest: Nothing to report. 
the alimentary tract. The elimination of the gastric acid barrier facilitates intestinal microbiota dysbiosis, causing bacterial overgrowth. Different studies have shown that PPIs, by altering the gut microbiota, may increase the occurrence of spontaneous bacterial peritonitis ${ }^{(18,19)}$ and other bacterial infections in patients with cirrhosis. ${ }^{(20)}$ A recent American study demonstrated that PPIs modulate readmission risk and microbiota composition in cirrhosis, which respond to withdrawal. ${ }^{(21)}$ Based on the relationship between PPIs, gut microbiota, and HE, three recent studies have investigated the correlation between PPI use and the risk of overt $\mathrm{HE}$ in patients with cirrhosis and found a significant association. ${ }^{(22-24)}$ Given their retrospective nature ${ }^{(22,23)}$ or the use of a population-based registry, ${ }^{(24)}$ in none of these studies was the presence of minimal HE (MHE) detected.

Actually, up to $60 \%$ of patients with cirrhosis are affected by a peculiar type of mild cognitive impairment regarding the selective attention and executive functions, visuomotor ability, psychomotor speed, response inhibition, and response selection that can be detected only by psychometry. ${ }^{(25,26)}$ The term minimal hepatic encephalopathy is used to indicate the presence of such subclinical cognitive alterations in patients who appear absolutely normal at clinical examination. Although subclinical, MHE has been shown to have important clinical implications, being associated with the development of overt $\mathrm{HE},{ }^{(27)}$ lower survival, ${ }^{(28)}$ and lower quality of life. ${ }^{(29)}$ Moreover, car accidents ${ }^{(30)}$ and falls ${ }^{(31)}$ are more frequent in patients with MHE. The alteration in gut microbiota may be implicated also in the occurrence of MHE. In particular, Gupta et al. demonstrated a higher prevalence of small intestine bacterial overgrowth in patients with cirrhosis and MHE. ${ }^{(32)}$ Thus, PPIs being associated with small intestine bacterial overgrowth and bacterial translocation ${ }^{(33)}$ may be implicated in the genesis of
MHE and its consequences, but no study has investigated the link between these drugs and MHE. Moreover, the prolonged use of high-dose PPIs has been associated with increased mortality in older patients discharged from acute-care hospitals ${ }^{(34)}$ as well as in a group of patients with cirrhosis. ${ }^{(35)}$

Given this background, we hypothesized that the chronic use of PPIs could be associated with the presence of MHE. For this aim we analyzed our database, in which a cohort of patients with cirrhosis prospectively enrolled and followed up was administered the Psychometric Hepatic Encephalopathy Score (PHES), which is considered the gold standard for the diagnosis of MHE, at entry and followed up to establish the incidence of overt $\mathrm{HE}$ and patient survival. A number of multivariate analyses were performed to establish the independent role of PPIs in the occurrence of $\mathrm{MHE}$ as well as in the development of overt $\mathrm{HE}$ and survival.

\section{Patients and Methods}

From January 2014 to August 2016, all patients with cirrhosis, both inpatients and outpatients, without overt HE admitted to the Center for the Study of Portal Hypertension in Rome were prospectively enrolled and their data included into a database aimed at establishing the prevalence of $\mathrm{MHE}$ and its risk factors. The diagnosis of liver cirrhosis was based on clinical, biochemical, and radiological signs. Clinical and biochemical characteristics and a detailed pharmacological treatment were collected for each patient. In the present study, the database was used specifically to analyze the role of PPIs on MHE and overt HE. At entry, overt HE was excluded by using a set of standardized closed questions based on the West-Haven criteria. ${ }^{(36)}$ Further exclusion criteria were alcohol/

\section{ARTICLE INFORMATION:}

From the ${ }^{1}$ Department of Clinical Medicine; ${ }^{2}$ Department of Public Health and Infectious Diseases, "Sapienza" University of Rome, Rome, Italy.

\section{ADDRESS CORRESPONDENCE AND REPRINT REQUESTS TO:}

Silvia Nardelli, M.D.

Department of Clinical Medicine

"Sapienza" University of Rome
Viale dell'Università 37

00161 Rome, Italy

Email: nardelli.silvia@gmail.com 
psychoactive drug intake (positive alcoholemia and/or benzodiazepines or opioid urine metabolites) at the moment of the psychometric evaluation, unrelated neurological disease, and lack of compliance with psychometric evaluation because of language barriers or reduced visual acuity. The presence of dementia was also excluded by using the Mini-Mental State Examination, as described. ${ }^{(37)}$ Patients with advanced hepatocellular carcinoma, outside the Milan criteria, were also excluded because in these patients the prognosis is strongly influenced by neoplastic disease. Patients with transjugular intrahepatic portosystemic shunts and/or large portosystemic shunts and patients with a history of persistent or recurrent $\mathrm{HE}$ defined by two or more episodes within the last 6 months, even if without overt $\mathrm{HE}$ on first observation, were also excluded. A detailed clinical history was obtained in relation to previous complications of liver cirrhosis, particularly previous episodes of overt HE. The patients were qualified as having a positive history if a previous episode of overt $\mathrm{HE}$ grade II or above (according to the West Haven criteria) was documented by a previous hospitalization. All of the other parameters (Child-Pugh class and score, Model for End-Stage Liver Disease [MELD] score, serum sodium and albumin levels) were collected during the enrollment hospitalization.

Informed, written consent for the collection and evaluation of demographic and clinical data was obtained. The "Sapienza" University of Rome Ethical Committee approved the study and allowed collection of the data (Rif.1720/01.10.09).

\section{PHARMACOLOGICAL ASSESSMENT}

Therapeutic regimens at baseline were carefully noted with regard to dose and duration of therapy. Information regarding drug exposure were retrieved from physician admission notes for inpatients and from medication lists in notes for outpatients. Patients were considered "PPI users" when the treatment started at least 4 weeks prior to admission. This timing was chosen according to pharmacodynamics and in keeping with the available literature. ${ }^{(38)}$ Regarding PPIs, we defined as "standard dosages" the daily administration of $20 \mathrm{mg}$ of omeprazole, $30 \mathrm{mg}$ of lansoprazole, and $40 \mathrm{mg}$ of pantoprazole or esomeprazole. ${ }^{(38)}$ We determined whether PPI treatment was given for strong indications (gastrointestinal bleeding, peptic ulcer disease, gastroesophageal reflux disease, endoscopic variceal ligation) or symptomatically for epigastric pain, nausea, or vomiting. To assess the duration of PPI therapy during follow-up, medical records were reviewed. At inclusion and during follow-up, only patients with a previous history of HE (25\%) took lactulose or nonabsorbable antibiotics or both. Some patients were on diuretics (57\%) or $\beta$-blockers (34\%). None of the patients took $\mathrm{H} 2$ blockers as acid-suppressive medications.

\section{EVALUATION OF MHE}

The psychometric evaluation was performed in a quiet room, with no distracting noises. All patients ( $\mathrm{n}=310$ ) underwent the PHES battery, including the digit-symbol test, the trail-making tests $\mathrm{A}$ and $\mathrm{B}$, the serial-dotting test, and the line-tracing test. ${ }^{(39)}$ Each test was scored against age-adjusted and educationadjusted norms for the Italian population. The PHES is the sum of integer scores of each test computed from the adjusted $Z$ values, as follows: score $=-3$ for $Z \leq-3$, score -2 for $-3<Z \leq-2$, score -1 for $-2<Z$ $\leq-1$, score 0 for $-1<Z<1$, score 1 for $Z \geq 1$. The PHES $\leq-4$ was considered abnormal. ${ }^{(40)}$ Moreover, to support the diagnosis of MHE, all patients underwent the animal naming test, which assesses the maximum number of animals listed in 1 minute, as described. ${ }^{(41)}$ The assessors of MHE were blinded to patients' pharmacological therapy.

\section{FOLLOW-UP}

All patients were offered follow-up in the outpatient department with repeated ultrasound and laboratory investigations every 6 months and endoscopic evaluation every 1 or 2 years. The patients and their families were instructed on the importance of adhering to the scheduled visits and to contact the medical staff immediately should any alteration in the mental status or neuromuscular function (especially asterixis and flapping tremor) occur between scheduled reviews. In particular, the family was instructed to report the occurrence of lethargy, apathy, obvious personality change, inappropriate behavior, or disorientation in time and place, which correspond to the occurrence of a grade II alteration in mental status. In this case, $\mathrm{HE}$ evaluation including the psychometric 
performance was repeated to confirm and stage the degree of HE. Patients with an overt episode of $\mathrm{HE}$ reached the main endpoint of the study. Patients were contacted by phone every 3 months to check on their adherence to the scheduled follow-up.

The patients were followed up until death, liver transplantation, or the last available outpatient review. During the whole follow-up none of the patients stopped PPI consumption.

\section{STATISTICAL ANALYSIS}

The data are reported as mean $\pm \mathrm{SD}$. Comparisons between groups were performed by an unpaired Student $t$ test or chi-squared test. Logistic regression analysis was used to identify clinical and biochemical variables independently and significantly associated with MHE. We estimated the cumulative incidence of the first episode of HE during the follow-up, taking into account the nature of the competing risks in the data (HE before liver transplantation, death, or liver transplantation are competing events) with the subdistribution model of Fine and Gray. The conditional subdistribution hazard at multivariate analysis was evaluated using the model of Fine and Gray. ${ }^{(42)}$ We therefore report on the subdistribution hazard ratios (HRs) rather than the usual HR, but the former have similar interpretations to the latter. The factors associated with the development of HE were initially evaluated by univariate models (using univariate Fine and Gray models) and then included in a multivariate analysis (according to multivariate Fine and Gray models). The Cox regression model was used to identify clinical and biochemical variables independently and significantly associated with mortality. The final multivariate models were chosen in a forward fashion by minimizing the Bayesian information criterion. Software R, version 3.4.2, was used for all computations. The analyses described above were repeated by excluding patients with previous episodes of overt $\mathrm{HE}$ in order to show the association between the analyzed variable in the subgroup of patients without previous HE.

Regarding the sample size calculation, assuming an overall risk of MHE of $50 \%$ and a relative risk of 1.5 for PPI users, a sample size of at least 263 patients guarantees a power of $90 \%$ to a chi-squared test at the $5 \%$ level for detecting an association between PPI and MHE.

\section{Results}

Of the 355 patients admitted to our department, some were excluded: 17 for the presence of overt $\mathrm{HE}$ (grade II or more), 4 for positive alcoholemia, 6 for psychoactive drug intake, 10 for altered Mini-Mental State Examination or neurological diseases, and 8 for lack of compliance with psychometric tests. Of the 310 patients enrolled, only 38 were inpatients, without signs of infections or acute decompensation of cirrhosis, admitted for elective procedures (prophylactic variceal band ligation, liver biopsy, locoregional treatments of early hepatocellular carcinoma). There were 207 patients considered affected by a decompensated disease (for the previous history of variceal bleeding or hepatic encephalopathy or the presence of ascites). The demographic, clinical, and biochemical characteristics of all patients enrolled in the study are reported in Table 1.

Of the 310 patients enrolled, 125 (40\%) were considered PPI users, as described. Within this class, the majority of the patients (80\%) were taking medications at a "standard dosage" for more than 12 months, and the remaining 25 patients (20\%) took PPIs for 3-12 months; none of the patients took PPIs for less than 3 months. The mean length of PPI use at baseline was $14.5 \pm 12.6$ months. In 52 of 125 patients the indications for PPI use were recent gastrointestinal

TABLE 1. Demographic and Clinical Characteristics of the Patients Enrolled in the Study*

Patients $(n=310)$

\section{$\operatorname{Sex}(M / F)$} $221 / 89$

Age (years) $62.2 \pm 11.8$

Etiology (virus/alcohol/other) $189 / 84 / 37$

MELD

$12.7 \pm 4.9$

Child-Pugh class $(A / B / C)$

$142 / 133 / 35$

Child-Pugh score

$7 \pm 1.7$

Previous HE (no/yes)

233/77

Ascites (no/yes)

$150 / 160$

Gastrointestinal bleeding (no/yes)

$225 / 85$

Decompensated cirrhosis (no/yes)

$103 / 207$

Hemoglobin $(\mathrm{g} / \mathrm{dL})$

$11.2 \pm 2.3$

Bilirubin (mg/dL)

$2.7 \pm 4.8$

Albumin $(\mathrm{g} / \mathrm{dL})$

$3.4 \pm 0.6$

International normalized ratio

$1.4 \pm 0.3$

Sodium (mEq/L)

$137 \pm 4.4$

*Values are mean \pm SD. 
bleeding, recent endoscopic ligation of varices, severe reflux, or peptic ulcer disease. In the remaining 73 patients PPIs were prescribed symptomatically for epigastric pain or abdominal discomfort, so an appropriate indication was lacking in a considerable number (58\%) of patients taking PPI therapy.

At the time of inclusion, PPI users and nonusers were similar in gender, age, severity of liver disease (expressed with MELD and Child-Pugh score), previous history of $\mathrm{HE}$, and presence of ascites. In PPI users serum sodium and albumin levels were significantly lower than those in nonusers. Moreover, the prevalence of MHE, diagnosed with PHES $\leq-4$, was significantly higher in PPI users than in nonusers (62\% versus $29 \%$; $P<0.001$ ) (Table 2 ). The prevalence of MHE in PPI users was higher than that in nonusers even when the 77 patients with previous overt $\mathrm{HE}$ were excluded from the analysis (PPI users, $\mathrm{n}=89, \mathrm{MHE}=57 \%$, versus PPI nonusers, $\mathrm{n}=144$, MHE 20\%; $P<0.001)$.

The comparison between patients with and without MHE is reported in Table 3. Patients with MHE, compared to those without, had a more severe stage of liver disease and a higher prevalence of overt $\mathrm{HE}$ in the past. Notably, in patients with MHE, more patients were taking PPI than in the group without MHE. The difference was maintained even when the

TABLE 2. Comparison of Demographic and Clinical Characteristics Between PPI Users and Nonusers

\begin{tabular}{lccc} 
& $\begin{array}{c}\mathrm{PPI}^{-} \\
(\mathrm{n}=185)\end{array}$ & $\begin{array}{c}\mathrm{PPI}^{+} \\
(\mathrm{n}=125)\end{array}$ & $P$ \\
\hline Sex (M/F) & $137 / 48$ & $84 / 41$ & $\mathrm{NS}$ \\
Age (years) & $61.5 \pm 11.9$ & $63.3 \pm 11.6$ & $\mathrm{NS}$ \\
Etiology (virus/alcohol/other) & $105 / 54 / 26$ & $84 / 30 / 11$ & $\mathrm{NS}$ \\
MELD & $12.3 \pm 4.5$ & $13.3 \pm 5.5$ & $\mathrm{NS}$ \\
Child-Pugh class (A/B/C) & $87 / 82 / 16$ & $55 / 51 / 19$ & $\mathrm{NS}$ \\
Child-Pugh score & $6.9 \pm 1.7$ & $7.2 \pm 1.8$ & $\mathrm{NS}$ \\
Previous HE (no/yes) & $144 / 41$ & $89 / 36$ & $\mathrm{NS}$ \\
Ascites (no/yes) & $92 / 93$ & $57 / 68$ & $\mathrm{NS}$ \\
Gastrointestinal bleeding (no/yes) & $138 / 47$ & $87 / 38$ & $\mathrm{NS}$ \\
Decompensated cirrhosis (no/yes) & $68 / 117$ & $35 / 90$ & $\mathrm{NS}$ \\
MHE (no/yes) & $131 / 54$ & $48 / 77$ & $<0.001$ \\
Hemoglobin (g/dL) & $11.1 \pm 2.3$ & $11.5 \pm 2.1$ & $\mathrm{NS}$ \\
Bilirubin (mg/dL) & $2.2 \pm 3.2$ & $3.3 \pm 6.4$ & 0.04 \\
Albumin (g/dL) & $3.4 \pm 0.6$ & $3.3 \pm 0.6$ & 0.21 \\
International normalized ratio & $1.3 \pm 0.3$ & $1.4 \pm 0.3$ & $\mathrm{NS}$ \\
Sodium (mEq/L) & $137.6 \pm 3.8$ & $136.1 \pm 5.1$ & 0.005
\end{tabular}

*Values are mean $\pm \mathrm{SD}$.

Abbreviation: NS, not significant.
77 patients with previous overt $\mathrm{HE}$ were excluded from the analysis $\left(\mathrm{MHE}^{+}, \mathrm{n}=80\right.$, PPI users $=64 \%$, versus $\mathrm{MHE}^{-}, \mathrm{n}=153$, PPI users $\left.25 \% ; P<0.001\right)$.

On multivariate analysis, including MELD scores, previous overt HE, PPIs, age, albumin, and sodium levels, the variables independently associated with the presence of MHE were PPIs, history of previous overt HE, albumin and sodium levels, and age (Table 4). The area under the receiver operating characteristic curve (AUROC) was 0.83. The significant relationship between PPI use and MHE was maintained when the multivariate analysis was repeated after exclusion of the 77 patients with overt $\mathrm{HE}$ in the past (PPI, odds ratio, 5.04; confidence interval [CI], 2.649.63; $P<0.001)$.

During a mean follow-up of $14.1 \pm 12.3$ months, 127 patients experienced grade II or higher HE. A precipitating factor was identified in 98 patients (77\%): 31 episodes (32\%) were caused by infections, $21(21.5 \%)$ by diuretic overdose and electrolyte disorders, 21 (21.5\%) by dehydration induced by vomiting and diarrhea, 13 (13\%) by gastrointestinal bleeding, 9 (9\%) by constipation, and 3 (3\%) by benzodiazepines. The incidence of HE, taking into account as a competitive risk death or liver transplantation, was significantly higher in PPI users than in nonusers $(64 \%$ versus $25 \%, P<0.001$ ) (Fig. 1 ).

TABLE 3. Comparison of Demographic and Clinical Characteristics Between Patients With and Without MHE

\begin{tabular}{lccc} 
& $\begin{array}{c}\text { MHE }^{-} \\
(n=179)\end{array}$ & $\begin{array}{c}\text { MHE }^{+} \\
(n=131)\end{array}$ & $P$ \\
\hline Sex (M/F) & $130 / 49$ & $91 / 40$ & NS \\
Age (years) & $62.2 \pm 11.6$ & $62.3 \pm 12.2$ & NS \\
Etiology (virus/alcohol/other) & $108 / 45 / 26$ & $81 / 39 / 11$ & NS \\
MELD & $12.1 \pm 4.7$ & $13.7 \pm 5.2$ & 0.005 \\
Child-Pugh class (A/B/C) & $99 / 68 / 12$ & $43 / 65 / 23$ & $<0.001$ \\
Child-Pugh score & $6.6 \pm 1.6$ & $7.6 \pm 1.8$ & $<0.001$ \\
Previous HE (no/yes) & $153 / 26$ & $80 / 51$ & $<0.001$ \\
Ascites (no/yes) & $106 / 73$ & $43 / 88$ & $<0.001$ \\
Decompensated cirrhosis (no/yes) & $74 / 105$ & $29 / 102$ & $<0.001$ \\
PPIs (no/yes) & $131 / 48$ & $54 / 77$ & $<0.001$ \\
Bilirubin (mg/dL) & $2.2 \pm 3.7$ & $3.2 \pm 5.9$ & NS \\
Albumin (g/dL) & $3.5 \pm 0.6$ & $3.2 \pm 0.6$ & $<0.001$ \\
International normalized ratio & $1.3 \pm 0.3$ & $1.4 \pm 0.3$ & NS \\
Sodium (mEq/L) & $138.1 \pm 3.6$ & $135.7 \pm 5.1$ & $<0.001$ \\
Animal naming test (no. & $16.4 \pm 5.4$ & $12.9 \pm 4.7$ & $<0.001$ \\
$\quad$ of animals) & & &
\end{tabular}

*Values are mean $\pm \mathrm{SD}$.

Abbreviation: NS, not significant. 
TABLE 4. Results of Multivariate Analyses

A. Results of the Logistic Regression Analysis Predicting the Presence of MHE

\begin{tabular}{|c|c|c|c|}
\hline Patients $(n=310)$ & OR & $\mathrm{Cl}$ & $P$ \\
\hline PPI & 3.96 & $2.27-6.92$ & $<0.001$ \\
\hline Previous overt HE & 3.38 & $1.74-6.57$ & $<0.001$ \\
\hline Sodium & 0.90 & $0.84-0.97$ & 0.006 \\
\hline Albumin & 0.37 & $0.22-0.62$ & $<0.001$ \\
\hline Age & 1.03 & $0.99-1.05$ & 0.055 \\
\hline MELD score & 0.97 & $0.91-1.03$ & 0.44 \\
\hline \multicolumn{4}{|c|}{ B. Results of the Competitive Risk Analysis (Fine and Gray Model) Predicting the Occurrence of Overt HE } \\
\hline & Subdistribution HR & $\mathrm{Cl}$ & $P$ \\
\hline PPI & 1.83 & $1.22-2.74$ & 0.003 \\
\hline Previous overt HE & 2.45 & 1.66-3.58 & $<0.001$ \\
\hline Albumin & 0.47 & 0.33-0.69 & $<0.001$ \\
\hline MHE & 1.79 & $1.21-2.65$ & 0.003 \\
\hline Age & 1.01 & 0.99-1.02 & 0.21 \\
\hline MELD score & 1.01 & $0.96-1.06$ & 0.63 \\
\hline Sodium & 0.98 & $0.95-1.02$ & 0.55 \\
\hline \multicolumn{4}{|l|}{ C. Results of the Cox Regression Analysis Predicting the Mortality } \\
\hline & HR & $\mathrm{Cl}$ & $P$ \\
\hline PPI & 2.37 & $1.45-3.87$ & $<0.001$ \\
\hline Age & 1.03 & $1.02-1.06$ & $<0.001$ \\
\hline Sodium & 0.93 & $0.89-0.97$ & $<0.001$ \\
\hline MELD score & 1.10 & $1.06-1.15$ & $<0.001$ \\
\hline Albumin & 0.64 & 0.43-0.95 & 0.03 \\
\hline MHE & 1.53 & $0.97-2.42$ & 0.03 \\
\hline Development of overt HE & 1.82 & $1.09-3.01$ & 0.01 \\
\hline Previous HE & 1.02 & $0.98-1.04$ & 0.21 \\
\hline
\end{tabular}

On multivariate analysis, including MELD score, previous overt HE, MHE (diagnosed with PHES $\leq-4$ ), PPIs, age, albumin and sodium levels; the variables independently associated with the development of overt HE were PPIs, history of overt HE, albumin levels, and MHE (Table 4). The AUROC was 0.78. The significant relationship between PPI use and the development of overt $\mathrm{HE}$ during the follow-up was maintained when the multivariate analysis was repeated after exclusion of the 77 patients with overt $\mathrm{HE}$ in the past (PPI, subdistribution $\mathrm{HR}=2.44$; CI 1.35-4,39; $P=0.003$ ).

During the same follow-up, 7 patients $(2 \%)$ were lost to follow-up, 16 (5\%) underwent liver transplantation, and 108 (35\%) died. The main causes of death were infections and sepsis (36\%), liver failure (33\%), variceal bleeding $(12 \%)$, and other causes not related to liver disease (19\%).
Overall survival was significantly lower in PPI users than in nonusers (41\% versus $81 \%, P<0.001$ ) (Fig. 2). On multivariate analysis, including MELD score, PPI use, previous history of $\mathrm{HE}$, development of overt $\mathrm{HE}, \mathrm{MHE}$, age, and albumin and sodium levels, the variables independently associated with mortality were PPIs, age, sodium and albumin levels, MELD score, presence of MHE, and development of overt HE (Table 4). The AUROC was 0.82. The significant relationship between PPI use and mortality was maintained when the multivariate analysis was repeated after exclusion of the 77 patients with overt HE in the past (PPI, HR, 2.08; CI 1.22-4.11; $P=0.0003)$.

The same results were obtained including ChildPugh score instead of MELD score in each multivariate analysis (data not shown). 


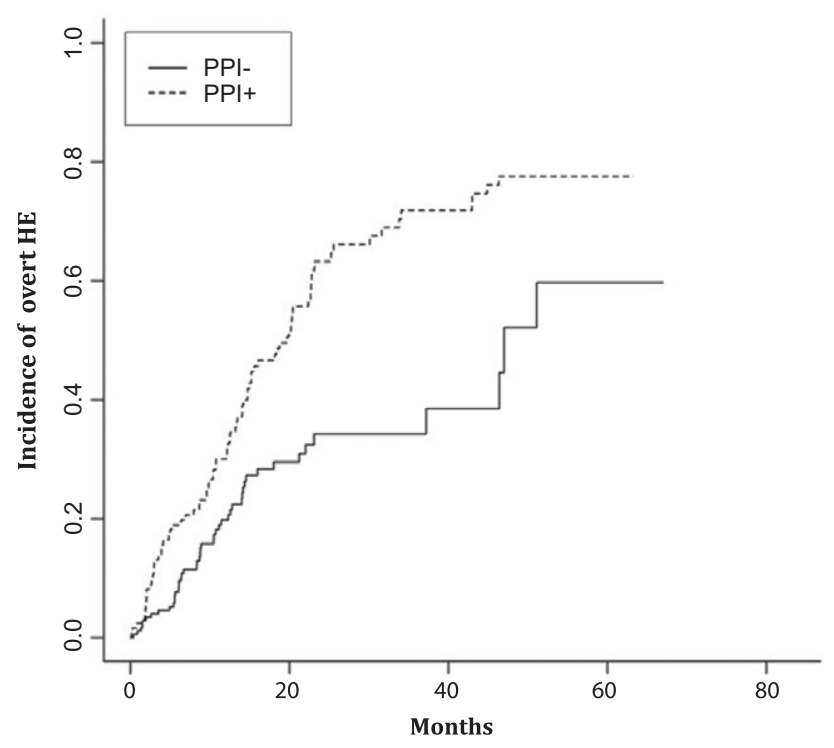

FIG. 1. Cumulative incidence of overt $\mathrm{HE}$ among PPI users and nonusers.

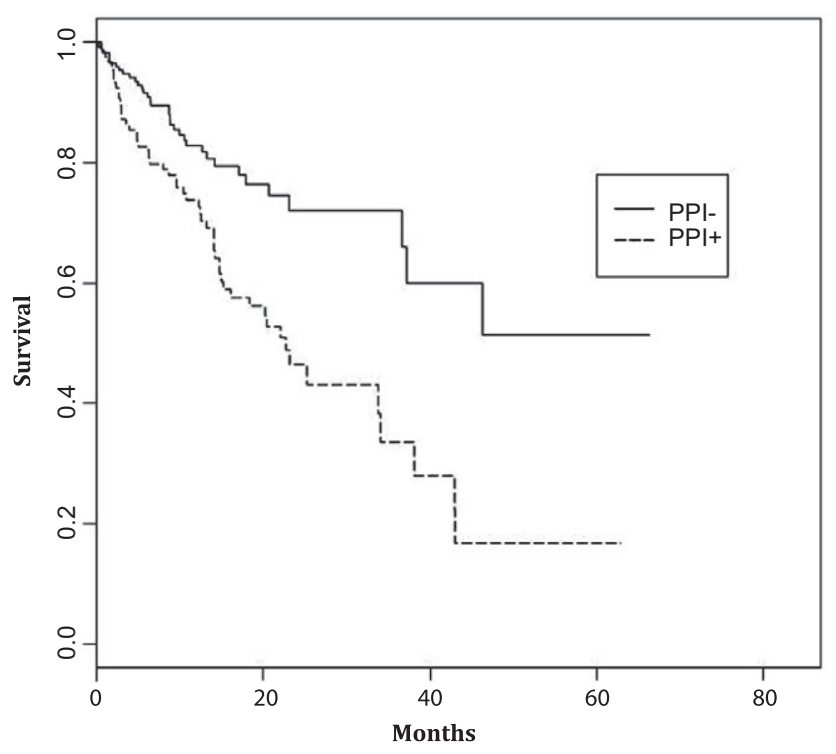

FIG. 2. Overall survival among PPI users and nonusers.

\section{Discussion}

The study showed that in patients with cirrhosis the use of PPIs is associated with the presence of MHE. The study also confirmed that PPI use is associated with an increased risk of overt $\mathrm{HE}^{(22-24)}$ and that both PPI use and the development of overt HE during follow-up increase mortality independently on very important prognostic factors such as age and MELD.

In our study, MHE, detected by the animal naming test and PHES, which is considered the gold standard for the diagnosis of this complication, was much more prevalent in PPI users than in nonusers $(62 \%$ versus 29\%); and on multivariate logistic analysis, PPI use was associated with the presence of MHE independently of the degree of liver failure and history of overt HE. It has been shown that a cognitive impairment may persist even after a single episode of overt $\mathrm{HE}^{(43,44))}$; thus, the association between PPI use and $\mathrm{MHE}$, independently on a strong risk factor such as the history of overt HE, strengthens the importance of PPI use as a risk factor for the presence of cognitive impairment in patients with cirrhosis.

As far as the relationship between PPIs and overt $\mathrm{HE}$, three studies have shown such a statistical association, ${ }^{(22-24)}$ which was confirmed also in our series. In fact, during follow-up, the cumulative incidence of overt HE, taking into consideration orthotopic liver transplantation and death as possible competing risks, was significantly higher in PPI users than in nonusers and was independent of well-known risk factors such as previous overt HE. Because $\mathrm{HE}$ is a recurrent complication of liver cirrhosis, patients with previous episodes of overt $\mathrm{HE}$ are particularly at risk of having further episodes during follow-up. However, the association between PPI use and development of overt HE during follow-up was maintained even when the patients with previous overt $\mathrm{HE}$ were excluded.

A limitation recognized in the previous studies showing a relationship between PPIs and overt $\mathrm{HE}$ is represented by the fact that the patients were not tested for the presence of MHE. MHE is a wellknown risk factor ${ }^{(27)}$ for the development of overt HE; thus, a relationship between PPIs and HE should be tested taking into consideration the confounding role of MHE. In our study PPI use was significantly associated with overt $\mathrm{HE}$ independently of the presence of $\mathrm{MHE}$ and of previous episodes of overt $\mathrm{HE}$, which is another important risk factor for the development of overt HE.

Finally, PPI use was associated with mortality independently of overt HE, age, and MELD score. The data relating PPI use and mortality in patients with cirrhosis are limited and controversial, ${ }^{(35,45,46)}$ being associated with increased mortality only in two studies out of three. 
In our series, $40 \%$ of patients with cirrhosis were using PPIs, and this treatment was prolonged for several months in the totality of the patients. These results are in line with previous reports on the very common use of PPIs, often inappropriately, in patients with cirrhosis. ${ }^{(47,48)}$

All of these observations strongly suggest great caution in using PPIs in patients with cirrhosis and support greater attention in limiting the use of PPIs to their strict indication and for a limited time. Actually, PPIs are often overused in patients with cirrhosis. ${ }^{(47)}$ In fact, they are often prolonged after an episode of variceal bleeding or band ligation, when their use is appropriate only for less than 2 months. ${ }^{(48)}$ Moreover, PPIs are frequently prescribed for a generic gastroprotection or because of dyspeptic symptoms. In our series only $42 \%$ of patients received PPI with an acid-related indication such as ulcer, reflux disease, or esophagitis.

Gastric acid is a defense mechanism against ingested microorganisms; thus, the reduction of gastric acidity by PPIs, by increasing the bacterial proliferation in the stomach and small intestine, may predispose to bacterial infections. ${ }^{(19,20,38)}$ Patients with cirrhosis receiving acid-suppression therapy are also at increased risk of being colonized by multidrug resistance bacteria. ${ }^{(35)}$ Finally, in advanced cirrhosis PPI metabolism may be impaired, and this can result in a higher exposure. ${ }^{(47)}$ All of these mechanism may induce bacterial overgrowth and translocation, thus exposing the patients to a low-grade inflammation, ${ }^{(32)}$ which has been related to $\mathrm{HE}$ as well as to the occurrence of infections. ${ }^{(38)}$

The main point of interest of the present study is the identification of a factor associated with MHE. MHE is an important complication of liver cirrhosis, being associated not only with the development of overt $\mathrm{HE}^{(27)}$ but also with the possible occurrence of car accidents, ${ }^{(30)}$ falls, ${ }^{(31)}$ low quality of life, ${ }^{(29)}$ and even low economic income. ${ }^{(49)}$ Despite its clinical relevance, the characteristics of patients with cirrhosis affected by this complication are still poorly known. We have previously described that MHE is more frequent in patients with cirrhosis and bacterial infections and that the cognitive impairment may be reversible after resolution of the infections. ${ }^{(50)}$ Herein we describe another potentially avoidable or suspendible factor associated with MHE, although the demonstration of the amelioration of MHE after the interruption of PPI use is lacking and not derivable by the present analysis.

The main limitation of the present study is inherent to the nature of our analysis. In fact, our results are based on statistical associations and not on pathophysiological data. Although PPI use maintained its independent role when submitted to complex statistical analysis which took into consideration the main identified factors associated with both the development of $\mathrm{HE}$ and mortality, we cannot exclude that other factors not considered or not identified may play the role attributed to PPI use, although a number of hypotheses may relate PPI use to the outcomes described herein. The amelioration of the cognitive impairment after PPI withdrawal may add further information, which is unfortunately lacking in the present study. Another limitation of the study is that the exposure of interest (PPI) was measured at the same time as the outcome (MHE); thus, at least for MHE, this can be considered a cross-sectional study.

In summary, PPI use was associated with an increased risk for mortality in a large cohort of patients with cirrhosis. It was an additional risk factor together with the stage of cirrhosis, hepatic decompensation, hepatocellular carcinoma, and infectious complications. Although a causative role for PPIs in the increased mortality cannot and should not be deduced from our observations, we advise careful use of PPIs in patients with cirrhosis given the potential adverse effects of PPIs, especially when they are used apart from hard indications for symptomatic treatment of abdominal symptoms.

Moreover, we have shown that prolonged use of PPIs is associated with the presence of MHE and with an increased risk for overt $\mathrm{HE}$ and mortality in a large cohort of patients with cirrhosis. Although a causative role for PPIs in the increased mortality cannot be deduced from our observations, we recommend careful prescription of PPIs in patients with cirrhosis, especially when they are used without specific indications.

\section{REFERENCES}

1) Vilstrup H, Amodio P, Bajaj J, Cordoba J, Ferenci P, Mullen $\mathrm{KD}$, et al. practice guideline by the American Association for the Study of Liver Diseases and the European Association for the Study of the Liver. Hepatology 2014;60:715-735.

2) Rai R, Saraswat VA, Dhiman RK. Gut microbiota: its role in hepatic encephalopathy. J Clin Exp Hepatol 2015;5(Suppl. 1):S29-S36. 
3) Bajaj JS, Heuman DM, Hylemon PB, Sanyal AJ, White MB, Monteith $\mathrm{P}$, et al. Altered profile of human gut microbiome is associated with cirrhosis and its complications. J Hepatol 2014;60:940-947.

4) Bajaj JS. Review article: potential mechanisms of action of rifaximin in the management of hepatic encephalopathy and other complications of cirrhosis. Aliment Pharmacol Ther 2016;43(Suppl. 1):11-26.

5) Bajaj JS, Heuman DM, Sanyal AJ, Hylemon PB, Sterling RK, Stravitz RT, et al. Modulation of the metabiome by rifaximin in patients with cirrhosis and minimal hepatic encephalopathy. PLoS One 2013;8:e60042.

6) Sharma BC, Sharma P, Lunia MK, Srivastava S, Goyal R, Sarin SK. A randomized, double-blind, controlled trial comparing rifaximin plus lactulose with lactulose alone in treatment of overt hepatic encephalopathy. Am J Gastroenterol 2013;108:1458-1463.

7) Agrawal A, Sharma BC, Sharma P, Sarin SK. Secondary prophylaxis of hepatic encephalopathy in cirrhosis: an open-label, randomized controlled trial of lactulose, probiotics, and no therapy. Am J Gastroenterol 2012;107:1043-1050.

8) Dhiman RK, Rana B, Agrawal S, Garg A, Chopra M, Thumburu KK, et al. Probiotic VSL\#3 reduces liver disease severity and hospitalization in patients with cirrhosis: a randomized, controlled trial. Gastroenterology 2014;147:1327-1337.

9) Sharma BC, Singh J. Probiotics in management of hepatic encephalopathy. Metab Brain Dis 2016;31:1295-1301.

10) Bajaj JS, Kassam Z, Fagan A, Gavis EA, Liu E, Cox IJ, et al. Fecal microbiota transplant from a rational stool donor improves hepatic encephalopathy: a randomized clinical trial. Hepatology 2017;66:1727-1738

11) Kao D, Roach B, Park H, Hotte N, Madsen K, Bain V, et al. Fecal microbiota transplantation in the management of hepatic encephalopathy. Hepatology 2016;63:339-340.

12) Scarpignato C, Gatta L, Zullo A, Blandizzi C; SIF-AIGOFIMMG Group, Italian Society of Pharmacology, et al. Effective and safe proton pump inhibitor therapy in acid-related diseases-a position paper addressing benefits and potential harms of acid suppression. BMC Med 2016;14:179.

13) Bajaj JS, Cox IJ, Betrapally NS, Heuman DM, Schubert ML, Ratneswaran M, et al. Systems biology analysis of omeprazole therapy in cirrhosis demonstrates significant shifts in gut microbiota composition and function. Am J Physiol Gastrointest Liver Physiol 2014;307:G951-G957.

14) Freedberg DE, Toussaint NC, Chen SP, Ratner AJ, Whittier S, Wang TC, et al. Proton pump inhibitors alter specific taxa in the human gastrointestinal microbiome: a crossover trial. Gastroenterology 2015;149:883-885.

15) Imhann F, Bonder MJ, Vich Vila A, Fu J, Mujagic Z, Vork L, et al. Proton pump inhibitors affect the gut microbiome. Gut 2016;65:740-748.

16) Jackson MA, Goodrich JK, Maxan ME, Freedberg DE, Abrams JA, Poole AC, et al. Proton pump inhibitors alter the composition of the gut microbiota. Gut 2016;65:749-756.

17) Vesper BJ, Jawdi A, Altman KW, Haines GK 3rd, Tao L, Radosevich JA. The effect of proton pump inhibitors on the human microbiota. Curr Drug Metab 2009;10:84-89.

18) Min YW, Lim KS, Min BH, Gwak GY, Paik YH, Choi MS, et al. Proton pump inhibitor use significantly increases the risk of spontaneous bacterial peritonitis in 1965 patients with cirrhosis and ascites: a propensity score matched cohort study. Aliment Pharmacol Ther 2014;40:695-704.

19) Miozzo SA, Tovo CV, John JA, de Mattos AA. Proton pump inhibitor use and spontaneous bacterial peritonitis in cirrhosis: an undesirable association? J Hepatol 2015;63:529-530.
20) Bajaj JS, Ratliff SM, Heuman DM, Lapane KL. Proton pump inhibitors are associated with a high rate of serious infections in veterans with decompensated cirrhosis. Aliment Pharmacol Ther 2012;36:866-874.

21) Bajaj JS, Acharya C, Fagan A, White MB, Gavis E, Heuman $\mathrm{DM}$, et al. Proton pump inhibitor initiation and withdrawal affects gut microbiota and readmission risk in cirrhosis. Am J Gastroenterol 2018;113:1177-1186.

22) Lin $\mathrm{ZN}$, Zuo YQ, Hu P. Association of proton pump inhibitor therapy with hepatic encephalopathy in hepatitis B virus-related acute-on-chronic liver failure. Hepat Mon 2014;14:e16258.

23) Dam G, Vilstrup H, Watson H, Jepsen P. Proton pump inhibitors as a risk factor for hepatic encephalopathy and spontaneous bacterial peritonitis in patients with cirrhosis with ascites. Hepatology 2016;64:1265-1272.

24) Tsai CF, Chen MH, Wang YP, Chu CJ, Huang YH, Lin $\mathrm{HC}$, et al. Proton pump inhibitors increase risk for hepatic encephalopathy in patients with cirrhosis in a population study. Gastroenterology 2017;152:134-141.

25) Weissenborn K, Giewekemeyer K, Heidenreich S, Bokemeyer $\mathrm{M}$, Berding G, Ahl B. Attention, memory, and cognitive function in hepatic encephalopathy. Metab Brain Dis 2005;20: 359-367.

26) Amodio P, Del Piccolo F, Marchetti P, Angeli P, Iemmolo R, Caregaro L, et al. Clinical features and survival of cirrhotic patients with subclinical cognitive alterations detected by the number connection test and computerized psychometric tests. Hepatology 1999;29:1662-1667.

27) Riggio O, Amodio P, Farcomeni A, Merli M, Nardelli S, Pasquale C, et al. A model for predicting development of overt hepatic encephalopathy in patients with cirrhosis. Clin Gastroenterol Hepatol 2015;13:1346-1352.

28) Ampuero J, Simón M, Montoliú C, Jover R, Serra MA, Cordoba $\mathrm{J}$, et al. Minimal hepatic encephalopathy and critical flicker frequency are associated with survival of patients with cirrhosis. Gastroenterology 2015;149:1483-1489.

29) Bianchi G, Giovagnoli M, Sasdelli AS, Marchesini G. Hepatic encephalopathy and health-related quality of life. Clin Liver Dis 2012;16:159-170.

30) Bajaj JS. Minimal hepatic encephalopathy matters in daily life. World J Gastroenterol 2008;14:3609-3615.

31) Román E, Córdoba J, Torrens M, Torras X, Villanueva C, Vargas $\mathrm{V}$, et al. Minimal hepatic encephalopathy is associated with falls. Am J Gastroenterol 2011;106:476-482.

32) Gupta A, Dhiman RK, Kumari S, Rana S, Agarwal R, Duseja $A$, et al. Role of small intestinal bacterial overgrowth and delayed gastrointestinal transit time in cirrhotic patients with minimal hepatic encephalopathy. J Hepatol 2010;53:849-855.

33) Lewis SJ, Franco S, Young G, O'Keefe SJ. Altered bowel function and duodenal bacterial overgrowth in patients treated with omeprazole. Aliment Pharmacol Ther 1996;10:557-561.

34) Maggio M, Corsonello A, Ceda GP, Cattabiani C, Lauretani F, Butto V, et al. Proton pump inhibitors and risk of 1-year mortality and rehospitalization in older patients discharged from acute care hospitals. JAMA Intern Med 2013;173:518-523.

35) Dultz G, Piiper A, Zeuzem S, Kronenberger B, Waldmann O. Proton pump inhibitor treatment is associated with the severity of liver disease and increased mortality in patients with cirrhosis. Aliment Pharmacol Ther 2015;41:459-466.

36) Conn HO, Leevy CM, Vlahcevic ZR, Rodgers JB, Maddrey $\mathrm{WC}$, Seeff $\mathrm{L}$, et al. Comparison of lactulose and neomycin in the treatment of chronic portal-systemic encephalopathy. A double blind controlled trial. Gastroenterology 1977;72:573-583.

37) Nardelli S, Pentassuglio I, Pasquale C, Ridola L, Moscucci F, Merli M, et al. Depression, anxiety and alexithymia symptoms 
are major determinants of health related quality of life (HRQoL) in cirrhotic patients. Metab Brain Dis 2013;28:239-243.

38) Merli M, Lucidi C, Di Gregorio V, Giannelli V, Giusto M, Ceccarelli G, et al. The chronic use of beta-blockers and proton pump inhibitors may affect the rate of bacterial infections in cirrhosis. Liver Int 2015;35:362-369.

39) Weissenborn K, Ennen JC, Schomerus H, Ruckert N, Hecker H Neuropsychological characterization of hepatic encephalopathy. J Hepatol 2001;34:768-773.

40) Amodio P, Campagna F, Olianas S, Iannizzi P, Mapelli D, Penzo M, et al. Detection of minimal hepatic encephalopathy: normalization and optimization of the Psychometric Hepatic Encephalopathy Score. A neuropsychological and quantified EEG study. J Hepatol 2008;49:346-353.

41) Campagna F, Montagnese S, Ridola L, Senzolo M, Schiff S, De Rui M, et al. The animal naming test: an easy tool for the assessment of hepatic encephalopathy. HePATology 2017;66:198-208

42) Fine JP, Gray RJ. A proportional hazards model for the subdistribution of a competing risk. J Am Stat Assoc 1999;94:496-509.

43) BajajJS, Schubert CM, Heuman DM, Wade JB, Gibson DP, Topaz A, et al. Persistence of cognitive impairment after resolution of overt hepatic encephalopathy. Gastroenterology 2010;138:2332-2340.

44) Riggio O, Ridola L, Pasquale C, Nardelli S, Pentassuglio I, Moscucci F, et al. Evidence of persistent cognitive impairment after resolution of overt hepatic encephalopathy. Clin Gastroenterol Hepatol 2011;9:181-183.

45) Kwon JH, Koh S, Kim W, Jung YJ, Kim JW, Kim BG, et al. Mortality associated with proton pump inhibitors in cirrhotic patients with spontaneous bacterial peritonitis. J Gastroenterol Hepatol 2014;29:775-781.

46) de Vos M, De Vroey B, Garcia BG, Roy C, Kidd F, Henrion $\mathrm{J}$, et al. Role of proton pump inhibitors in the occurrence and the prognosis of spontaneous bacterial peritonitis in cirrhotic patients with ascites. Liver Int 2013;33:1316-1323.

47) Kalaitzakis E, Bjornsson E. Inadequate use of proton-pump inhibitors in patients with liver cirrhosis. Eur J Gastroenterol Hepatol 2008;20:512-518.

48) Chavez-Tapia NC, Tellez-Avila FI, Garcia-Leiva J, Valdovinos MA. Use and overuse of proton pump inhibitors in cirrhotic patients. Med Sci Monit 2008;14:CR48-CR48.

49) Bajaj JS, Riggio O, Allampati S, Prakash R, Gioia S, Onori E, et al. Cognitive dysfunction is associated with poor socioeconomic status in patients with cirrhosis: an international multicenter study. Clin Gastroenterol Hepatol 2013;11:1511-1516.

50) Merli M, Lucidi C, Pentassuglio I, Giannelli V, Giusto M, Di Gregorio V, et al. Increased risk of cognitive impairment in cirrhotic patients with bacterial infections. J Hepatol 2013;59:243-250. 